\title{
Transferts d'électrons à longue distance dans les protéines
}

Les transferts d'électrons intramoléculaires à longue distance, qui peuvent se produire au sein des protéines, joueraient un rôle important dans les processus biologiques d'oxydo-réduction dont l'efficacité serait ainsi accrue.

\section{Moshe Faraggi Christiane Ferradini Jean-Paul Jay-Gerin}

\section{ADRESSES}

M. Faraggi : Professeur. Département de chimie, centre de recherche nucléaire-Negev, BP 9001, Beer-Sheva, Israè1.

C. Ferradini : Professeur. Laboratoire de chimie-physique, université René-Descartes, 45, rue des Saints-Pères, 75270 Paris Cedex 06, France.

J.-P. Jay-Gerin: Professeur. Groupe du conseil d e recherches médicales du Canada en sciences des radiations et département de médecine nucléaire et de radiobiologie, faculté de médecine, université de Sherbrooke, Sherbrooke, Québec, Canada JlH l est bien connu que l'oxygène peut se réduire facilement en eau selon le processus global d'oxydo-réduction :

$\mathrm{O}_{2}+4 \mathrm{H}^{+}+4 \mathrm{e}^{-} \rightarrow 2 \mathrm{H}_{2} 0$ (l)

$\mathrm{A}$ cette réduction de l'oxygène peut correspondre, pour les organismes vivants aérobies, l'oxydation de molécules biologiques (substrats). Toutefois, ces réactions de l'oxygène sont le plus souvent très lentes et demandent donc à être catalysées. Tel est le cas de la phosphorylation oxydative (respiration), au cours de laquelle les quatre électrons impliqués dans la réaction (1) sont échangés un par un (figure 1). Cette chaîne est constituée par divers transporteurs d'électrons, tels que NAD (nicotinamide adénine dinucléotide), FAD (flavine adénine dinucléotide), CoQ (coenzyme $Q$ ) et divers cytochromes. Le potentiel d'oxydoréduction ( $\left.\mathrm{E}^{\prime} \mathrm{o}\right)$ diminue le long de la chaîne permettant ainsi aux électrons en provenance du substrat de réduire l'oxygène en eau [1]. Selon un mécanisme habituellement admis, le transfert d'électrons s'effectue au moment où le groupement réactionnel du donneur et celui de l'accepteur sont «en contact» ( $\lesssim 5 \AA$ ). Pour que la réaction se poursuive entre partenaires, il est nécessaire pour les protéines, et donc dans le cas particulier précédent pour les cytochromes, que s'établisse momentanément une déformation, c'est-à-dire un changement de conformation (effet allostérique) (figure 2).

Selon un autre mécanisme, le trans- fert électronique peut impliquer la migration d'un électron sur une distance supérieure à $5 \AA$ à travers la protéine. Une telle possibilité a été proposée dès 1965 par Winfield [2] pour le cytochrome $c$. Cette intuition a été depuis confirmée à l'aide de méthodes de cinétique rapide, telles que la radiolyse pulsée et la photolyse éclair [3].

\section{Principes des méthodes de cinétique rapide}

Les techniques de radiolyse pulsée et de photolyse éclair consistent à produire en solution des espèces radicalaires, ou bien des espèces excitées ou photoionisées. Ces espèces sont formées par des impulsions brèves $\left(10^{-6}\right.$ à $10^{-9} \mathrm{~s}$ ) d'électrons de haute énergie dans le cas de la radiolyse pulsée et de photons (UV et visible) dans le cas de la photolyse éclair. L'interaction des électrons de haute énergie s'effectue avec les molécules du solvant, tandis que les photons interagissent directement au niveau du soluté. L'évolution des réactions est suivie par spectrophotométrie résolue dans le temps. Il est ainsi possible de détecter et d'identifier les intermédiaires réactionnels (figure 3 ).

\section{Résultats actuels concernant les transferts d'électrons à longue distance}

L'existence de transferts intramoléculaires d'électrons à grande distance a maintenant été mise en évidence 

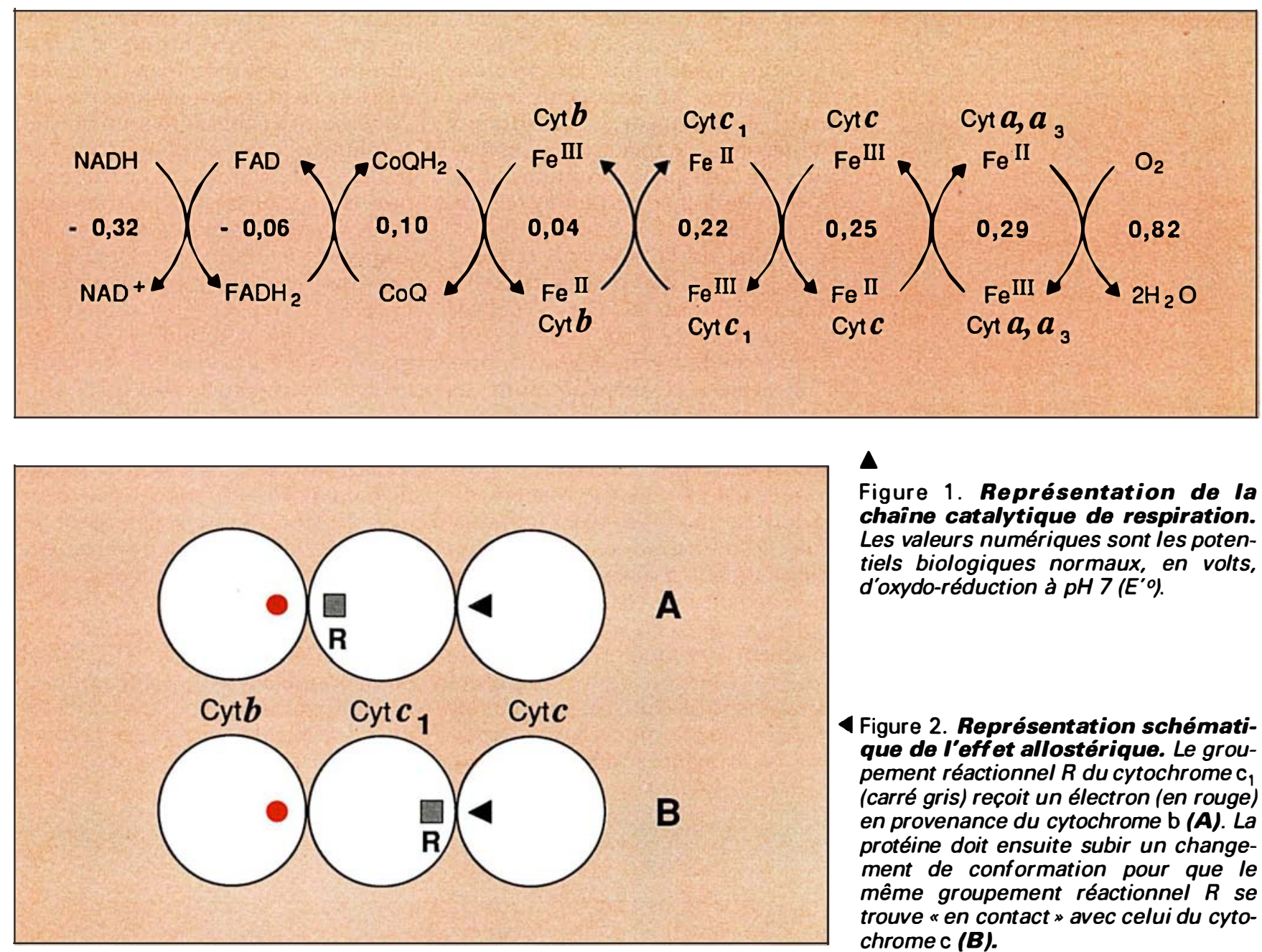

Figure 1. Représentation de la chaîne catalytique de respiration. Les valeurs numériques sont les potentiels biologiques normaux, en volts, d'oxydo-réduction à $\mathrm{pH} 7\left(E^{\prime} \%\right)$.

4 Figure 2. Représentation schématique de l'effet allostérique. Le groupement réactionnel $R$ du cytochrome $\mathrm{c}_{1}$ (carré gris) reçoit un électron (en rouge) en provenance du cytochrome b (A). La protéine doit ensuite subir un changement de conformation pour que le même groupement réactionnel $R$ se trouve « en contact avec celui du cytochrome c (B).

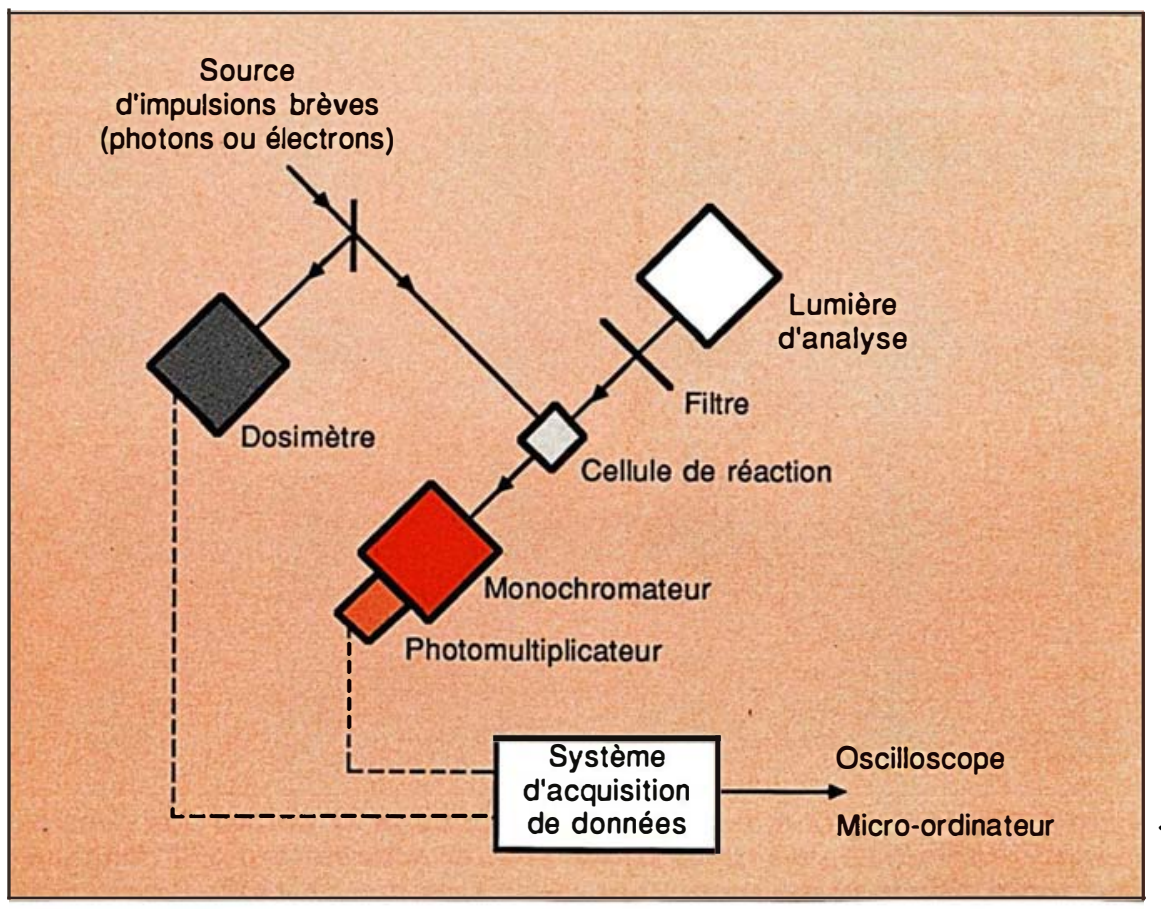

4 Figure 3. Schéma de montages de radiolyse pulsée et de photolyse 


\section{RÉFÉRENCES}

1. Kruh J. Biochimie, vol. II. Paris: Hermann, 1983.

2. Winfield ME. Electron transfer within and between haemoprotein molecules. J Mol Biol $1965 ; 12: 600-11$.

3. Hammes GG. Techniques of Chemistry, vol. 6. New York: Wiley-Interscience, 1974.

4. McLendon G, Miller JR. The dependence of biological electron transfer rates on exothermicity: the cytochrome $c /$ cytochrome $b_{5}$ couple. J Am Chem Soc 1985 ; 107 : 7811-6.

5. Simolo KP, McLendon GL, Mauk MR, MaukAG. Photoinduced electron transfer within a protein-protein complex formed bet ween physiological redox partners : reduction of ferricytochrome $b_{5}$ by the hemoglobin derivative $\alpha_{2}{ }^{\mathrm{Zn}} \beta_{2} \mathrm{Fe}^{\mathrm{III}} \mathrm{CN}$. J Am Chem Soc 1984; $106: 5012-3$.

6. McLendon G. Long-distance electron transfer in proteins and model systems. $A c c$ Chem Res $1988 ; 21$ : 160-7.

7. Faraggi M, Klapper MH. Intramolecular long-range electron transfer in the $\alpha$-hemoglobin subunit. J Am Chem Soc 1988 ; 110 : 5753-6.

8. McLendon G, Pardue K, Bak P. Electron transfer in the cytochrome $c /$ cytochrome $b_{2}$ complex: evidence for «conformational gating». J Am Chem Soc 1987 ; 109 : 7540-1.

9. Peterson-Kennedy SE, McGourty JL, Hoff man BM. Temperature dependence of long range electron transfer in [ $\left.\mathrm{Zn}, \mathrm{Fe}^{\mathrm{III}}\right]$ hybrid hemoglobin. J Am Chem Soc 1984; 106 : 5010-2.

10. Isied SS Kuehn C Worosila G Ruthe nium-modified cytochrome $c$ : temperature dependence of the rate of intramolecular electron transfer. J Am Chem Soc 1984; 106 1722-6.

10 bis. Nocera DG, Winkler JR, Yocom KM Bordignon E, Gray HB. Kinetics of intramolecular electron transfer from $\mathrm{Ru}^{\mathrm{II}}$ et $\mathrm{Fe}^{\mathrm{III}}$ in ruthenium-modified cytochrome c. J Am Chem Soc $1984 ; 106$ : 5145-50. pour une vingtaine de protéines. Comme on pouvait s'y attendre, ces réactions suivent une loi cinétique $\mathrm{du} \mathrm{l}^{\mathrm{er}}$ ordre. Le paramètre le plus étudié actuellement est la distance qui sépare le donneur de l'accepteur, mais est également examinée l'influence de la nature physico-chimique de l'environnement concerné, de la nature, de la structure et de la configuration des centres d'oxydoréduction avant et après l'échange d'électron, de la force motrice de la réaction de transfert, définie comme étant égale à la différence entre les potentiels biologiques normaux d'oxydo-réduction du couple oxydant et du couple réducteur.

Pour de telles études, la protéine a dû parfois être modifiée par l'introduction d'un second couple oxydoréducteur, af in d'obtenir un donneur et un accepteur présents sur la même molécule tout en connaissant les paramètres évoqués. Dans d'autres cas, au contraire, sont examinés directement certains constituants de la chaîne de phosphorylation oxydative, constituants formés par l'asso- ciation de deux protéines telles que le complexe cytochrome b/cytochrome $c$. Les principaux résultats décrits à ce jour sont résumés dans le Tableau I. Ce tableau montre que les constantes de vitesse des réactions de transferts d'électrons $(k)$ varient dans une large gamme $\left(10^{7}\right)$, diminuant grosso modo lorsque la distance $(d)$ augmente et croissant lorsque la force motrice, $\Delta E^{\prime}$, croît. Signalons, toutefois, que ces constantes de vitesse semblent diminuer pour les grandes valeurs de $\Delta \mathrm{E}^{\prime}$. Ce comportement serait dans le sens de la plupart des théories actuellement développées concernant les transferts d'électrons (voir ci-dessous). L'examen du Tableau I montre également que de tels transferts paraissent se produire suivant une seule direction. En effet, si on compare les expériences concernant le cytochrome $c$ modifié, on voit que le fait de remplacer le couple $\mathrm{Ru}^{\mathrm{II}} / \mathrm{Fe}^{\mathrm{III}}[10,10 \mathrm{bis}]$ par le couple $\mathrm{Fe}^{\mathrm{II}} / \mathrm{Ru}^{\mathrm{III}}$ [14] inhibe la réaction au lieu de l'inverser. De plus, le rôle privilégié du cytochrome $c$ comme médiateur de transfert

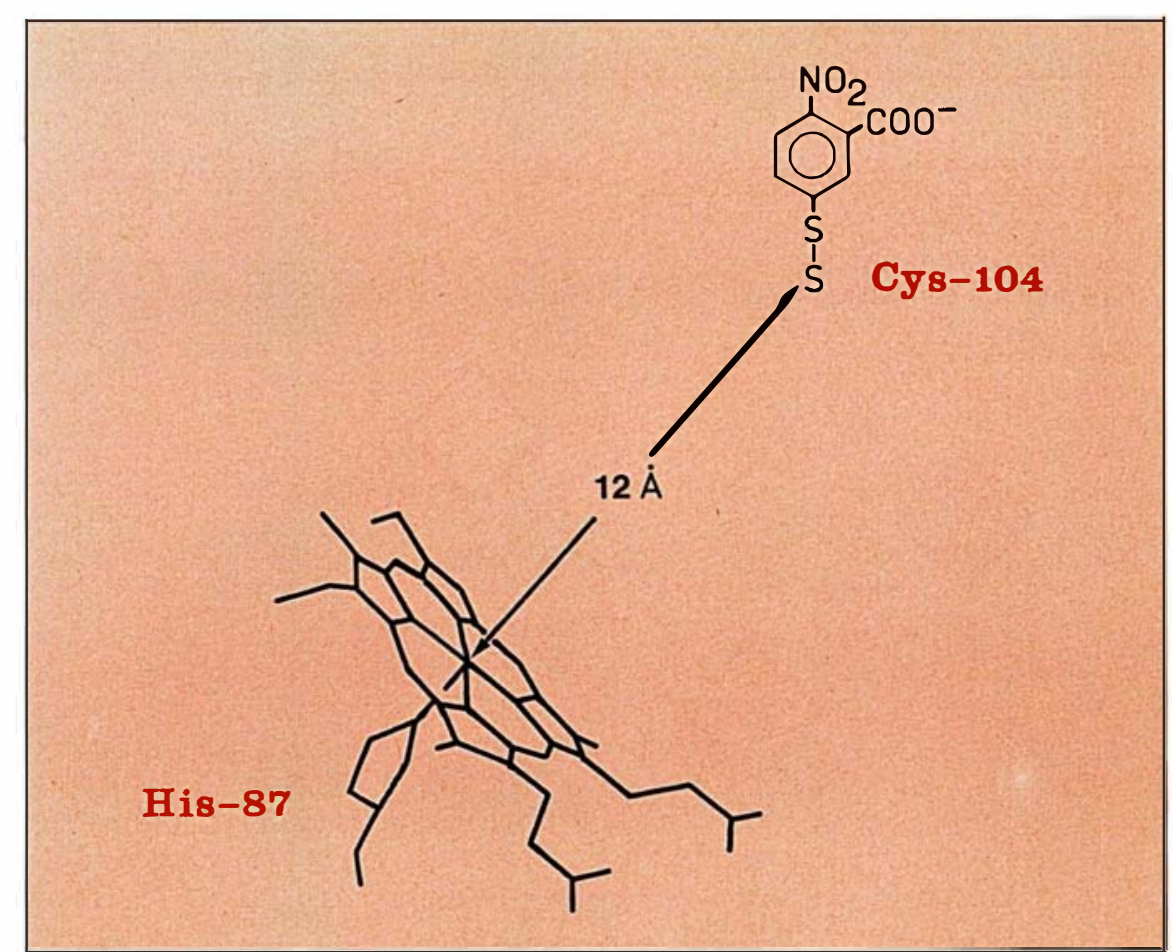

Figure 4. Représentation, à titre d'exemple, de la structure des centres donneur (thionitrobenzoate-cystéine 104) et accepteur (Fe'il) dans une sous-unité $\alpha$ de la méthémoglobine (d'après Fermi et al. [15]). 
Tableau 1

CONSTANTES DE VITESSE DES RÉACTIONS INTRAMOLÉCULAIRES

\begin{tabular}{|c|c|c|c|c|c|}
\hline Donneur/Accepteur & Référence & Système & $\Delta E^{\circ} \circ(V)^{a}$ & $d(A))^{b}$ & $k\left(s^{-1}\right)^{c}$ \\
\hline $\mathrm{Zn} \|^{\prime *} / \mathrm{Fe}^{\| \prime \prime}$ & 4 & Complexe cyt $c$ modifié/cyt $b_{5}$ & 0,8 & 8 & $2 \times 10^{5}$ \\
\hline $\mathrm{H}_{2}$ porph $* / \mathrm{Fe}^{\prime \prime \prime}$ & 4 & Complexe apocyt $c /$ cyt $b_{5}$ & 0,4 & 8 & $5 \times 10^{4}$ \\
\hline $\mathrm{Zn}^{\prime \prime *} / \mathrm{Fe}$ & 5 & Complexe $\mathrm{Zn}-\mathrm{Hb}$ modifiée/cyt $b_{5}$ & 1,0 & 7 & $8 \times 10^{3}$ \\
\hline $\mathrm{H}_{2}$ porph $-/ \mathrm{Fe}^{\text {III }}$ & 4 & Complexe apocyt $c / c y t b_{5}$ & 1,1 & 8 & $8 \times 10^{3}$ \\
\hline $\mathrm{Fe} " / / \mathrm{Fe}{ }^{\prime \prime \prime}$ & 6 & Complexe cyt $b_{5} /$ cyt $c$ de bœuf & 0,2 & 8 & $6 \times 10^{3}$ \\
\hline $\mathrm{Fe}{ }^{\prime \prime} / \mathrm{Fe}^{\| \prime \prime}$ & 4 & Complexe cyt $b_{5} / c y t c$ de levure & 0,2 & 8 & $1,7 \times 10^{3}$ \\
\hline $\mathrm{S}-\mathrm{S}-/ \mathrm{Fe}^{\prime \prime \prime}$ & 7 & $\alpha$-Hémoglobine modifiée & 0,44 & 12 & $2 \times 10^{2}$ \\
\hline $\mathrm{Fe}^{\prime \prime} / \mathrm{Fe}^{\prime \prime \prime}$ & 8 & Complexe cyt $b_{2}$ de levure/cyt $c$ & 0,2 & 8 & $1,5 \times 10^{2}$ \\
\hline $\mathrm{H}_{2}$ porph $* / F e^{I I I}$ & 8 & Complexe apocyt $c / c y t b_{2}$ & 0,4 & 8 & $1,5 \times 10^{2}$ \\
\hline $\mathrm{Zn}{ }^{11 *} / \mathrm{Fe} \mathrm{e}^{\prime \prime \prime}$ & 8 & Complexe $\mathrm{Zn}$-cyt $c$ modifié $/ c y t ~ b_{2}$ & 0,8 & 8 & $1,5 \times 10^{2}$ \\
\hline$\alpha-\mathrm{Zn}{ }^{\prime \prime *} / \beta-\mathrm{Fe}^{\prime \prime \prime}$ & 9 & $\mathrm{Zn} / \mathrm{Fe}$-hémoglobine modifiée & 0,8 & 20 & $1,0 \times 10^{2}$ \\
\hline $\mathrm{Ru} " / \mathrm{Fe}{ }^{\prime \prime \prime}$ & 10 & Ru-cytochrome $c$ modifié & 0,15 & 12 & 40 \\
\hline $\mathrm{S}-\mathrm{S} \div / \mathrm{Fe}^{\prime \prime \prime}$ & 11 & Hémerythrine modifiée & 0,78 & 13 & 15 \\
\hline $\mathrm{Ru} " / \mathrm{Cu} \|$ & 12 & Ru-azurine modifiée & 0,24 & 12 & 2 \\
\hline $\mathrm{Ru}{ }^{\prime \prime} / \mathrm{Fe}{ }^{\prime \prime \prime}$ & 13 & Ru-cytochrome modifiée & $-0,02$ & 13 & $2 \times 10^{-2}$ \\
\hline $\mathrm{Fe} " / \mathrm{Ru}^{\prime \prime \prime}$ & 14 & Ru-cytochrome $c$ modifié & 0,18 & 12 & $<10^{-3}$ \\
\hline
\end{tabular}

a Force motrice de la réaction, exprimée en volts (déterminée par polarographie pulsée différentielle). b Distance bord-à-bord, exprimée en Angströms, entre les deux centres oxydo-réducteurs (déterminée par cristallographie aux rayons X). c Constante de vitesse, exprimée en inverse du temps en secondes, décrivant l'évolution exponentielle de la réaction.

d'électron est nettement confirmé par la différence de comportement des deux couples $\mathrm{Ru}^{\mathrm{II}} / \mathrm{Cu}^{\mathrm{II}}[12]$ (azurine modifiée) et $\mathrm{Ru}^{\mathrm{II}} / \mathrm{Fe}^{\mathrm{III}}[10,10$ bis] : bien que la force motrice de la première réaction soit supérieure à celle de la seconde, sa vitesse est 20 fois plus faible. Soulignons enfin que les transferts d'électrons concernant les couples $\mathrm{Fe}^{\mathrm{II}} / \mathrm{Fe}^{\mathrm{III}}$ dans le complexe cytochrome $b_{5}$ /cytochrome $c[4,6]$ et $\mathrm{S}-\mathrm{S} \% / \mathrm{Fe}^{\mathrm{III}}[7]$ dans l'a-hémoglobine modifiée (figure 4) se produisent au sein d'entités ayant une existence biologique réelle, tandis que les autres cas correspondent à des molécules modèles diverses.

\section{Théories des transferts d'électrons à longue distance}

Plusieurs modèles théoriques ont été avancés pour interpréter ces phénomènes nouveaux de transferts intramoléculaires d'électrons à longue distance au sein d'une protéine. La théorie classique de Marcus [16] a été établie initialement pour expliquer les réactions intermoléculaires de $\mathrm{m} / \mathrm{s} n^{\circ} 9 \mathrm{vol.4}$, novembre 88 transferts électroniques en solution. Elle suppose que le passage de l'électron survient lorsque le donneur et l'accepteur se trouvent « en contact ». Elle implique donc l'existence d'une interaction forte entre les espèces réagissantes. La figure 5 exprime la variation d'énergie potentielle du système en fonction d'une coordonnée réactionnelle. Sur cette figure, on voit que la valeur de $\mathrm{H}_{\mathrm{AD}}$ est d'autant plus grande que l'intensité de l'interaction précédente est importante. Pour les interactions fortes (figure $5 C$ ), lorsque l'arrangement des atomes est favorable (point F), la probabilité de transfert de l'électron est égale à l'unité (réaction dite adiabatique). C'est ainsi que la théorie de Marcus permet de distinguer la contribution de la réaction de transfert électronique proprement dite de l'ensemble des processus qui lui sont associés, tels que la polarisation du solvant, la solvatation, l'énergie de réorganisation du milieu, etc. Son développement prédit, en particulier, le fait que la constante de vitesse diminue lorsque la force motrice atteint des valeurs élevées (zone dite d'inversion de Marcus).

Pour les réactions de transferts intramoléculaires d'électrons, l'interaction est généralement faible par suite de la grande distance séparant le donneur de l'accepteur. La figure $5 B$ représente un tel exemple. Arrivé au point $F$, la faible valeur de $\mathrm{H}_{\mathrm{AD}}$ offre au système une alternative: soit de donner les produits, soit de ne pas quitter la courbe caractéristique des réactifs. La probabilité du transfert est alors inférieure à l'unité (réaction dite non-adiabatique). Cette probabilité a été calculée par Hopfield [17], Jortner [18] et d'autres auteurs [19, 19 bis] en utilisant les résultats de la mécanique quantique impliquant l'effet tunnel. Une description de cet effet est illustrée, d'une manière schématique, dans la figure 6. L'existence de deux zones de stabilité pour l'électron est représentée par deux puits de potentiel $P_{1}$ et $P_{2}$ séparés par une barrière. Le fait que l'électron, initialement en $P_{1}$, ait une longueur d'onde associée donne à celui-ci une probabilité de présence non nulle de se trouver en $\mathrm{P}_{2}$. La probabilité de passage de $\mathrm{P}_{1}$ en $\mathrm{P}_{2}$ est d'autant plus 


\section{RÉFÉRENCES}

12. KostićNM, MargalitR, Che C-M Gray HB. Kinetics of long-distance ruthenium-to-copper electron transfer in (pentaammineruthenium histidine-83) azurin. $J \mathrm{Am}$ Chem Soc 1983; 105 : 7765-7.

13. Crutchley R J, Ellis WR Jr, Gray HB. Long-distance electron transfer in pentaammineruthenium (histidine-48)-myoglobin Reorganizational energetics of a high-spin heme. J Am Chem Soc 1985 ; 107 : 5002-4.

14. Bechtold R, Kuehn C, Lepre C, Isied SS. Directional electron transfer in rutheniummodified horse heart cytochrome $c$. Nature $1986 ; 322$ : 286-8.

15. Fermig, PerutzMF, ShaananB, Fourme R. The crystal structure of human deoxyhemoglobin at $1.74 \AA$ resolution. $J \mathrm{Mol}$ Biol 1984 ; 175 : 159-14.

16. Marcus RA, Sutin N. Electron transfers in chemistry and biology. Biochim Biophys Acta 1985 ; 811 : 265-322.

17. Horpfield JJ. Electron transfer between biological molecules by thermally activated tunneling. Proc Natl Acad Sci USA 1974; 71 : $3640-4$.

18. Jortner J. Dynamics of the primary events in bacterial photosynthesis. $\mathrm{J} \mathrm{Am}$ Chem Soc $1980 ; 102$ : 6676-86.

19. Miller JR, Beitz JV. Long-range transfer of positive charge between dopant molecules in a rigid glassy matrix. $J$ Chem Phys 1981 ; $74: 6746-56$.

19 bis. Hush NS. Distance dependence of electron transfer rates. Coord Chem Rev 1985 . 64 : 135-57.

20. Pethig R. Dielectric and electronic properties of biological materials. Chichester : Wiley, 1979.

21. Jay-Gerin JP, Ferradini C, HouéeLevin C, Lopez-Castillo JM, Faraggi M. Long-range electron transfer in proteins: a percolation mechanism. Radiat Phys Chem $1987 ; 30: 309-10$.

22. Mayo SL, Ellis WR Jr, Crutchley RJ, Gray HB. Long-range electron transfer in 588
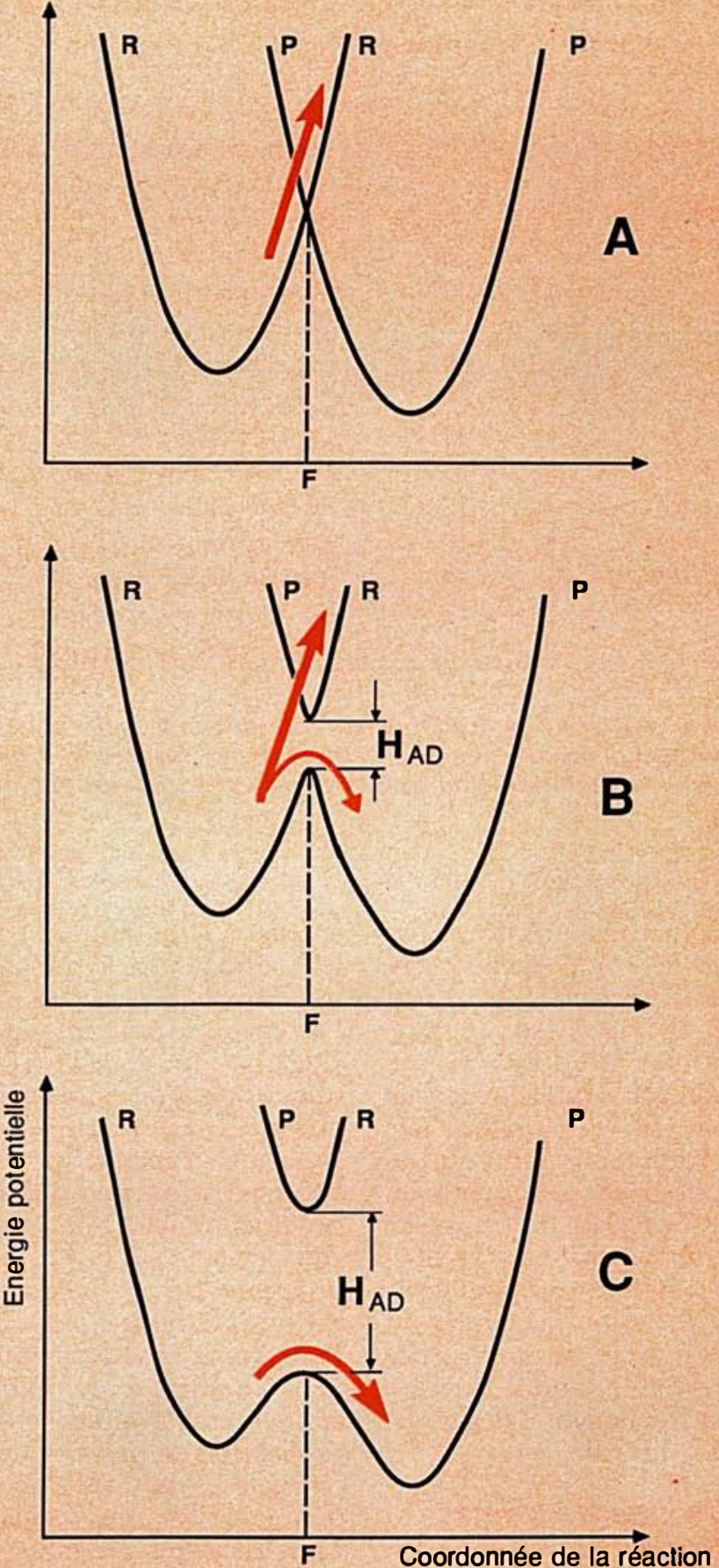

Figure 5. Illustration de l'influence des paramètres énergétiques intervenant dans les processus de transfert électronique. L'intensité de l'interaction entre donneur $(D)$ et accepteur $(A)$ est traduite par la longueur $H_{A O}$ correspondant à la coordonnée $F$ de la réaction. $P$ et $R$ désignent respectivement les courbes d'énergie potentielle des produits et des réactifs. $\boldsymbol{A}=$ absence d'interaction $\left(H_{A D}=0\right)$ et donc de transfert $; \boldsymbol{B}=$ interaction faible $; \boldsymbol{C}=$ interaction forte. 


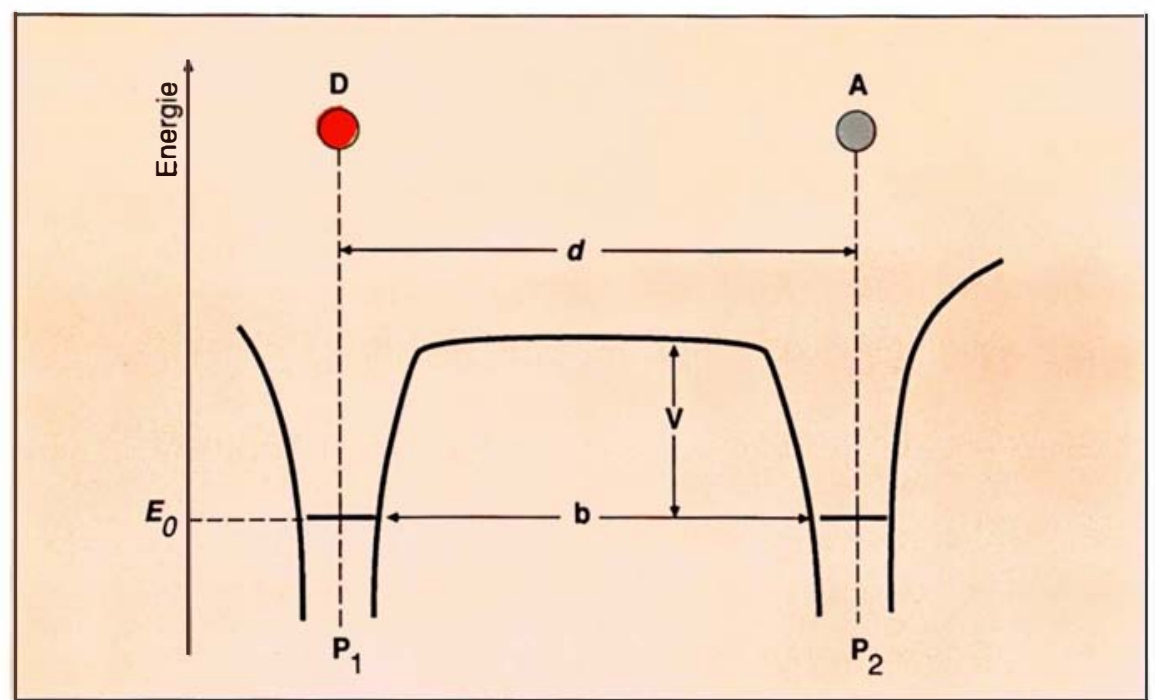

Figure 6. Illustration de l'effet tunnel. $d, V$ et $b$ désignent respectivement la distance entre le donneur $(D)$ (cercle rouge) et l'accepteur $(A)$ (cercle gris), la hauteur et la largeur de la barrière de potentiel. $E_{0}$ représente l'énergie de l'état lié de l'électron dans les deux puits, valeur pour laquelle le transfert s'effectue.

faible que la hauteur de la barrière est plus élevée. Les calculs ainsi réalisés ont permis, en particulier, d'établir la décroissance exponentielle de la constante de vitesse $k$ en fonction de la distance.

D'autres modèles ont également été proposés pour expliquer les transferts intramoléculaires d'électrons à longue distance dans les protéines. Citons ici le modèle introduit par Szent-Györgyi qui s'appuie sur des concepts de la théorie de l'état solide en établissant l'analogie entre les protéines et les macromolécules organiques possédant des propriétés semi-conductrices [20]. Cette analogie est fondée sur la présence de structures régulières répétitives, le «squelette » de la protéine. Mentionnons enfin le modèle de conductivité proposé récemment [21] qui s'appuie sur l'existence de conformations « conductrices » dans une protéine de nature isolante. Ce modèle est un exemple de percolation, ainsi nommé par analogie avec le brusque passage de l'eau bouillante dans le café en poudre contenu dans un percolateur. Les auteurs ont montré que la probabilité d'avoir une conformation favorable parmi l'ensemble des conformations possibles de la molécule est une fonction exponentielle de la longueur de la chaîne protéique. Ce dernier modèle aboutit, à ce point de vue, à des conclusions sem- blables à celles prédites par les théories impliquant l'effet tunnel.

\section{Perspectives}

Il apparaît que les résultats précédents ne peuvent pas être décrits par un mécanisme général ne prenant pas en considération la structure spécifique de la protéine. Ceci nous amène à considérer de nouvelles expériences pouvant employer des méthodes de génie génétique pour modifier la structure primaire de la protéine. Un lien plus étroit pourrait ainsi être établi entre la théorie et les expériences. Néanmoins, dans l'état actuel de nos connaissances, le phénomène de transfert d'électrons intramoléculaire à longue distance ouvre de nouvelles perspectives sur les mécanismes et l'énergétique des réactions d'oxydo-réduction biologiques. Il a d'ailleurs été émis l'idée que certaines protéines pourraient être considérées comme des «transférases d'électrons à longue distance» [22]. Le rôle de ces protéines consisterait à faciliter les transferts d'électrons in vivo et contribuerait ainsi au fait que les organismes vivants fonctionnent avec de très bons rendements énergétiques. Il est envisageable que, dans l'avenir, des processus artificiels fondés sur ce principe puissent in vitro s'avérer efficaces pour utiliser au mieux l'énergie

\section{Summary}

Recent investigations have established that various redox reactions can occur in proteins even if the electron-exchanging sites are separated by relatively large distances ( $10 \AA$ or more). These long-range electron transfers would be of central importance to many of the metabolic processes necessary for the survival of organisms. Although their mechanisms are still to be elucidated, it appears that they could lead to interesting applications. Research on biological electron transfer is one of current considerable activity. We briefly review here the results and recent developments in this area. 